\title{
Fresh washed platelets restore clotting time after extrinsic activation by ROTEM $\otimes$ reactants in an in vitro model for extreme Hemodilution
}

\author{
S. Pérez Garcia ${ }^{1}$, T. Koller ${ }^{1}$, P. Paniagua Iglesias ${ }^{1}$, G. Escolar Albaladejo², V. Moral Garcia ${ }^{1}$ \\ ${ }^{1}$ Hospital Santa Creu i Sant Pau - Barcelona (Spain), ${ }^{2}$ Hospital Clinic - Barcelona (Spain)
}

\section{Background and Goal of Study}

Clotting Time alterations in rotational thrombelastometry (Rotem®) caused by dilutional coagulopathy are usually interpreted as shortage of procoagulant plasmatic coagulation factors, limiting the potential of thrombin generation. Therefore current treatment algorhythms suggest factor substitution by FFP or prothrombin complex (PCC) transfusion. Although being a central element in the activation of coagulation factors the role of platelets on this thrombelastometrical parameter is not well studied. In an in vitro model for hemodilution whith exactly defined protein contents and free from blood cells we studied the influence of platelets on this important Rotem parameter.

\section{Materials and Methods}

- Stem solution (SS): Human albumin 5\% (Grifols, Spain) in Viaflo Plasmalyte ${ }^{\circ} 148$ (Baxter, Spain), enriched with Ca++ gluconate $(0,9 \mathrm{mmol} / \mathrm{l})$ was titrated with TRIS buffer $2 \mathrm{M}$ to $\mathrm{pH} 7,3-7,4$ and heated to $37^{\circ} \mathrm{C}$.

From this SS we create an enriched SS by adding:

- Fibrinogen concentrate (FC) to get a concentration of $4 \mathrm{~g} / \mathrm{L}$.

- Protrombin Complex Concentrate (PCC) to get a concentration of $1 \mathrm{IU} / \mathrm{ml}$.

- Factor thirteen (FXIII) was added to reach a concentration of $1 \mathrm{IU} / \mathrm{ml}$.

Starting from this protein enriched SS we analyzed 2 study samples:

1. Enriched SS without platelets

2. Enriched SS with platelets in a fixed concentration of 200.000 platelets/ $\mu$ l by adding the proper amount of washed platelets from a healthy donant.

The study samples were analyzed for viscoelastic properties with the EXTEM S and FIBTEM S subtest on a ROTEM® delta machine.

\section{Results and Discussion}

- $C T$ in study samples platelet free sample (mean value, $n=8): 128,13$.

- $C T$ in study samples with platelets (mean value, $n=5$ ): 73,60.

- A shortening in CT is observed in the platelet group. This shortening is statistically significant. $(p<0,001)$.

Figure 1 Restoring clotting time in an hemodilutional model after adding fresh washed platelets.

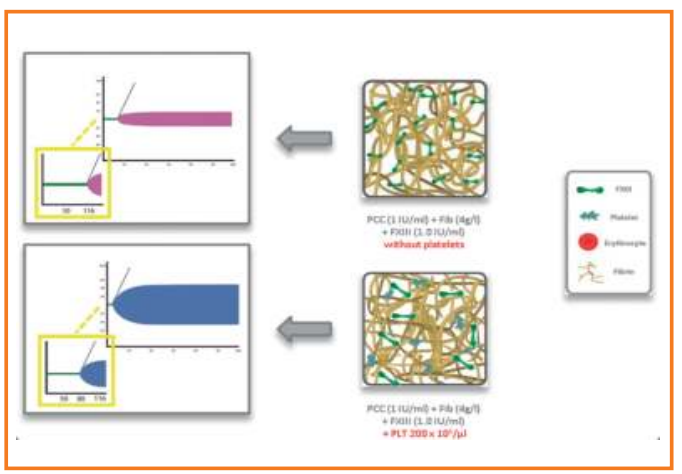

Figure 4. Box Plot comparing mean CT in both groups, after $t$ student test, the shorttening in $\mathrm{CT}$ in the platelet group is statistically significant $p<0.01$

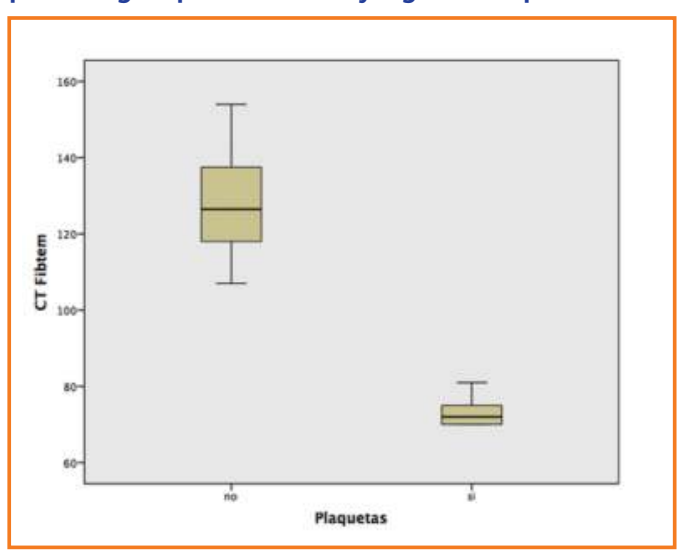

Figure 2. FIBTEM and EXTEM tests in our hemodilutional model

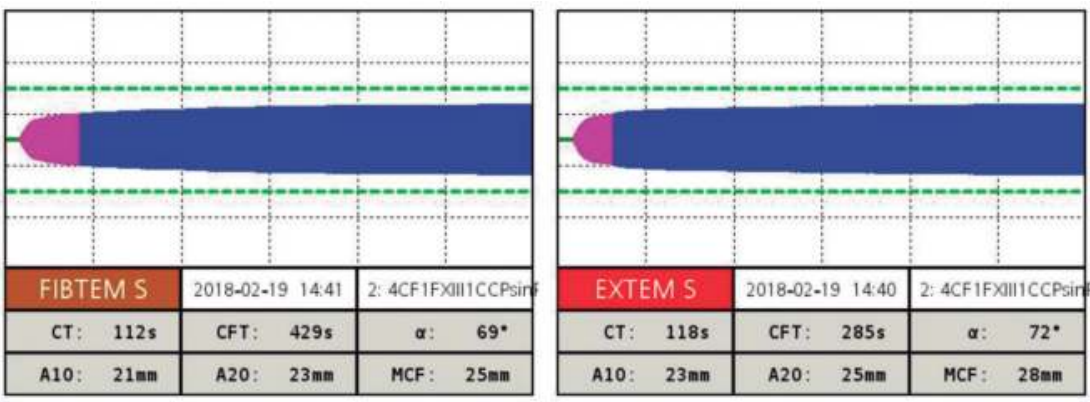

Figure 3. FIBTEM and EXTEM tests after adding to our hemodilutional model fresh washed platelets in a concentration of 200.000 platelets $/ \mu \mathrm{L}$.
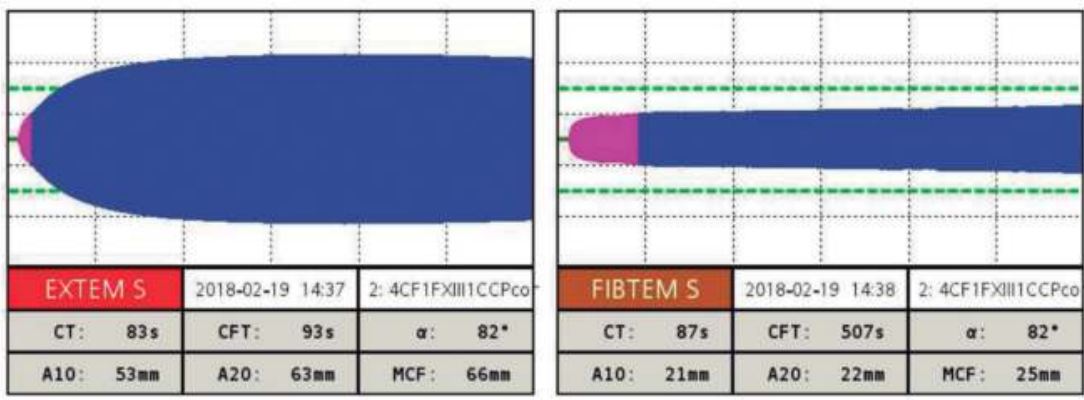

Conclusions

- In coagulopathic bleeding patients with prolonged CT values the transfusion of platelets (if indicated by clot firmness parameters) might improve or even correct this thrombelastometric treatment goal.

- This approach differs from usually proposed FFP or PCC treatment. 